\title{
A mismatch-tolerant RT-quantitative PCR: application to broad-spectrum detection of respiratory syncytial virus
}

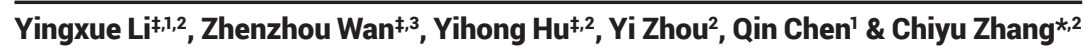

\section{ABSTRACT}

Quantitative PCR (qPCR) is widely used to detect viruses. However, mismatches occurring in the $3^{\prime}$-end of the primers reduce amplification efficiency of qPCR and limit its capacity in detection of highly variable viruses. Here, we reported a mismatchtolerant RT-qPCR with a small amount of additional high-fidelity DNA polymerase for simultaneous detection of RSV-A and RSV-B. The novel assay had higher amplification efficiency for various variants forming mismatches with the primers than the conventional RT-qPCR, and showed good specificity and sensitivity. It demonstrated a good correlation coefficient with a commercial RSV detection kit and had relatively lower $\mathrm{Ct}$ values than the kit for 16 of 20 RSV-positive samples. The mismatchtolerant qPCR technique is a promising approach for sensitive detection of highly variable viruses.

\section{METHOD SUMMARY}

We developed a mismatch-tolerant RT-quantitative PCR by simply adding a small amount of additional high-fidelity DNA polymerase to the standard reaction mixtures. The new method well tolerates mismatches between primers and templates and is especially suited to the detection of highly variable viruses.

\section{KEYWORDS:}

detection • high-fidelity DNA polymerase - mismatch-tolerant qPCR - respiratory syncytial virus $\cdot$ virus variant

'School of Life Sciences, Shanghai University, Shanghai, 200444, China; ${ }^{2}$ Pathogen Discovery \& Big Data Center, CAS Key Laboratory of Molecular Virology \& Immunology, Institut Pasteur of Shanghai, Chinese Academy of Sciences, Shanghai, 200031, China; ${ }^{3}$ Medical Laboratory of Taizhou Fourth People's Hospital, Taizhou, 225300, China; *Author for correspondence: zhangcy1999@ips.ac.cn

${ }^{\ddagger}$ Authors contributed equally to this study

BioTechniques 66: 225-230 (May 2019) 10.2144/ btn-2018-0184
Emerging and re-emerging infectious diseases seriously threaten global public health [1]. Most infectious diseases are caused by viruses, especially RNA viruses such as HIV-1, HCV, MERS-CoV, Ebola, Influenza A (H7N9) and Zika virus [2,3]. Rapid and accurate diagnosis of involved viruses is a pivotal step for the prevention and control of infectious diseases [1]. TaqMan probe-based real-time PCR was the most widely used and most robust method for qualitative and quantitative detection of viruses because of its high-sensitivity, closetube operation and real-time monitoring [4]. However, viruses are more genetically variable than prokaryotic and eukaryotic organisms [5,6]. In particular, RNA viruses (e.g., HIV-1 and HCV) have higher mutation rates than DNA viruses, and often evolve into various genotypes and subtypes $[7,8]$. It is relatively difficult to find a completely conserved genomic region shared by different genotypes and subtypes for PCR primer design. Therefore, degenerate primers were often used for pan-genotype/subtype detection of RNA viruses $[9,10]$. Despite this, the presence of various variants may cause mismatch between the primers and the targets. One or more mismatches occurring in the $3^{\prime}$ end of the primers will largely reduce amplification efficiency of qPCR [11], limiting the capacity of qPCR in the sensitive detection of highly variable viruses. Therefore, development of accurate, sensitive and broad-spectrum detection methods for highly variable viruses is still a big challenge.

Respiratory syncytial virus (RSV), an enveloped negative-strand RNA virus belonging to the genus Pneumovirus of the Paramyxoviridae family [12], is the main viral pathogen causing acute respiratory tract infections (ARTIs) in infants and young children [13-15]. RSV infection is associated with bronchiolitis, pneumonia, otitis media, rhinitis and other reactive airway diseases [16]. It has been estimated that there are approximately 33.1 million RSV-related cases worldwide per year in children younger than 5 years, and the mortality was $118,200[17,18]$. Early diagnosis and monitoring of RSV infection play a crucial role in the treatment and management of RSV patients.

RSV is genetically divided into two major distinct groups, RSV-A and RSV-B [19]. Although some detection methods, including serological and various nucleic acid amplification assays, have been developed [20-28], there still lacks an efficient RT-qPCR assay for simultaneous detection of all RSV-A and RSV-B variants. In this study, we developed a mismatchtolerant RT-qPCR technique with a small amount of additional high-fidelity DNA polymerase and used it to simultaneously detect various RSV-A and RSV-B variants.

\section{MATERIALS \& METHODS}

Reaction system of the mismatchtolerant RT-qPCR

Quant One Step RT-qPCR Kit (Tiangen Biotechnology Co., Ltd, Beijing, China) and Q5 high-fidelity DNA polymerase (New England Biolabs, USA) were used to establish the reaction system of the mismatch-tolerant RT-qPCR assay. The difference between the novel assay and the conventional TaqMan probe-based method is the addition of a small amount of high-fidelity DNA polymerase into the reaction system. The amount of Taq DNA polymerase and Q5 high-fidelity DNA polymerase was optimized at $0.75 \mathrm{U}$ and $0.15 \mathrm{U}$ per $25 \mu \mathrm{l}$ reaction mix, respec- $\downarrow$ 
(A) The conventional qPCR method
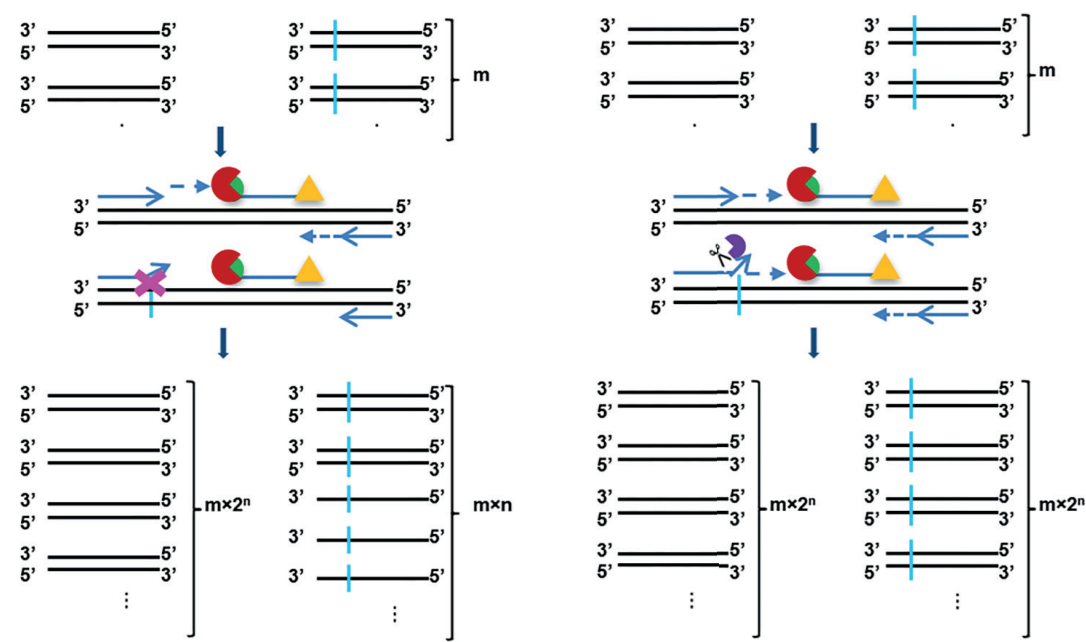

】

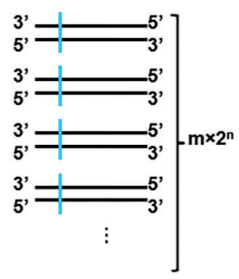

High fidelity DNA polymerase

Fluorescence group

Quenching group

Taq DNA polymerase Mutation

Figure 1. The principle of the mismatch-tolerant qPCR. (A) The principle of the conventional qPCR. The presence of a mismatch at the $3^{\prime}$ end of the primer will largely reduce or stop the amplification. (B) The principle of the mismatch-tolerant qPCR. When there is a mismatch between primer and target at the $3^{\prime}$ end of the primer, the small amount of high-fidelity DNA will remove the mismatched base from the primer by $3^{\prime}-5^{\prime}$ exonuclease activity, and then Taq DNA polymerase will initiate primer extension. In theory, mismatches between primer and target have less influence on DNA amplification by the mismatch-tolerant qPCR.

$\mathrm{M}$ : Copy numbers of templates; $n$ : PCR cycle number.

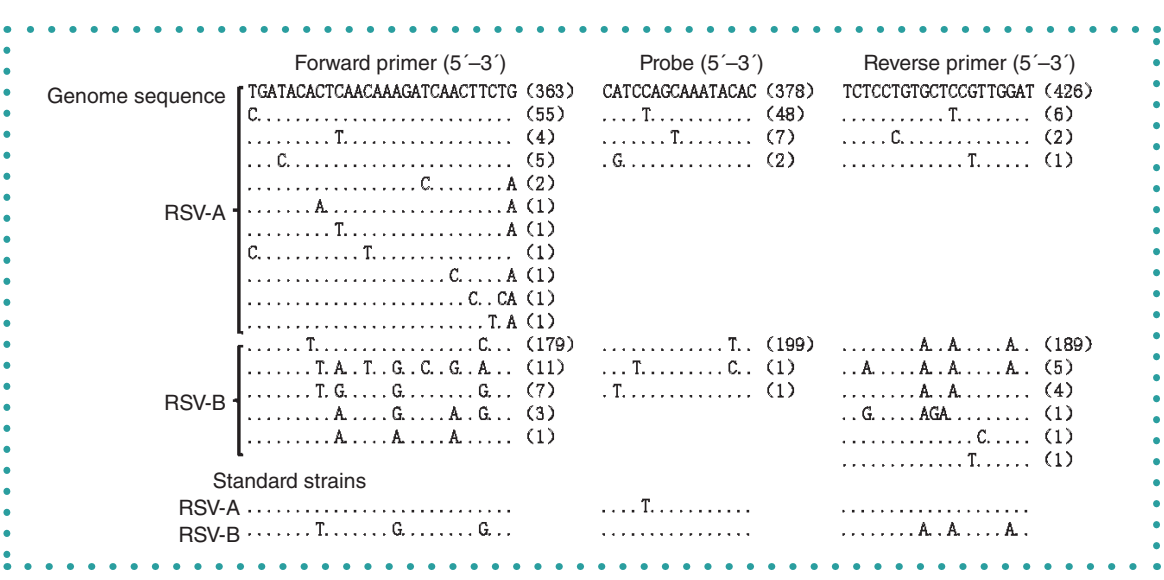

Figure 2. Sequence alignments of primer and probe regions of all available RSV sequences. A total of 636 sequences were downloaded from GenBank on June 8, 2018. The black small dots indicate identical bases to the topmost sequence. The numbers in the parenthesis show the number of identical sequences.

- tively. The optimized reaction mix of the novel assay included $12.5 \mu \mathrm{l} 2 \times$ Quant One Step Probe RT-qPCR Master Mix, $0.75 \mathrm{U}$ HotMaster Taq polymerase, 0.15U Q5 ${ }^{\circledR}$ Highfidelity DNA Polymerase (New England Biolabs, USA), $0.1 \mu$ l Quant RT, $400 \mathrm{nM}$ each forward and reverse primers, and $100 \mathrm{nM}$ RSV probe. The cycling condition was reverse transcription at $50^{\circ} \mathrm{C}$ for $30 \mathrm{~min}$, enzyme activation and denaturation at $92^{\circ} \mathrm{C}$ for $3 \mathrm{~min}$, then by 40 cycles of denaturation at $92^{\circ} \mathrm{C}$ for $10 \mathrm{~s}$, annealing and extension at $55^{\circ} \mathrm{C}$ for $40 \mathrm{~s}$. Fluorescence signal was collected at the annealing and extension step of each cycle. The threshold value was automatically set to 0.05 by the Light Cycler 96 Real-Time PCR System (Roche Diagnostics, Germany).

\section{Virus strains \& patient samples}

To determine the sensitivity and limit of detection (LOD) of the novel RSV RT-qPCR assay, two RSV standard strains, RSV-A (VR-1540) and RSV-B (VR-1400) were used. Eight standard respiratory virus strains, including adenovirus (VR-930), enterovirus (VR-1076), influenza $A$ and $B$ viruses (VR-333 and VR-789), parainfluenza viruses 3 (VR-93), HCoV-229E (VR-740), HCoV-OC43 (VR-1558), and human rhinovirus (VR-489) were used as control viruses to test the assay specificity. All the above strains were purchased from the American Type Culture Collection (ATCC) and stored in the Lab at Institut Pasteur of Shanghai, Chinese Academy of Sciences.

To evaluate the performance of the novel assay, 40 nasopharyngeal swabs collected from children with ARTIs in our previous studies were used $[29,30]$. Among these samples, half were previously detected as RSV-positive and half as RSV-negative using our in-house multiplex RT-qPCR method. The positive samples included 14 RSV-A and six RSV-B.

\section{RNA extraction \& preparation of RNA standards}

RNA was extracted from $140 \mu$ l virus culture supernatants or clinical samples and eluted in $50 \mu \mathrm{l}$ nuclease-free $\mathrm{H}_{2} \mathrm{O}$ by High Pure Viral RNA Kit (Roche Diagnostics $\mathrm{GmbH}$, Mannheim, Germany) according to the manufacturer's instruction. The RNA from $3.75 \times 10^{5.75} \mathrm{PFU} / \mathrm{ml}$ of RSV strain VR-1540 was used as a standard template to determine the LOD of the developed assay by serial tenfold dilution from $10^{5}$ to $10^{0} \mathrm{PFU} / \mathrm{ml}$.

\section{Construction of various mutants}

To test the performance of the novel assay for mismatched targets, we constructed a series of mutants that form mismatches with the RSV-specific primers. The targeted RSV fragment was obtained by amplification of the standard strain with a T7 promoter-containing primer and cloned into pMD18-T plasmid vector (TaKaRa, Dalian, China). A series of mutants were constructed using a rapid mutagenesis system of transgenic biotechnology (TransGen, Beijing, China), and confirmed by Sanger sequencing. Mutant RNA templates were prepared by in vitro transcription using T7 RNA polymerase. 
Evaluation of the assay using clinical samples

To assess the performance of the novel assay for RSV-A and RSV-B RNA detection, we evaluated it using 40 clinical samples and compared it with the conventional assay and a commercial kit (Huayin RSV RNA kit, Huayin Pharmaceutical Technology Co., Ltd, Guangdong, China). The kit was designed for detection of RSV-A and -B RNA and is often used in clinical RSV diagnosis in China. A Bland-Altman analysis was used to assess the level of agreement between the novel assay and the commercial kit. A range of agreement was defined as the mean of difference (bias) \pm 1.96 SD.

\section{RESULTS \& DISCUSSION \\ The principle of the mismatch-tolerant RT-qPCR}

Taq DNA polymerase that has 5'-3' exonuclease activity is widely used in conventional qPCR to emit a fluorescent signal by $5 '-3$ 'cleavage of the TaqMan probe. Because of the lack of $3^{\prime}-5^{\prime}$ exonuclease activity of the Taq DNA polymerase, mismatches between primers and templates, especially at the $3^{\prime}$ end of the primers, prevent primer extension or reduce the extension efficiency of PCR in the conventional method that uses Taq enzyme (Figure 1A). High-fidelity DNA polymerase that has a $3^{\prime}-5^{\prime}$ exonuclease activity can repair misincorporated bases and was previously used to detect mutation and quantify HIV-1 viral load, as well as gene expression [31-33]. To improve the applicability of the qPCR to variable templates, we developed a mismatch-tolerant RT-qPCR method by adding a small amount of highfidelity DNA polymerase into the reaction. When mismatches are formed between primers and templates, the small amount of high-fidelity DNA polymerase is triggered to recognize and remove the mismatched bases from the primers, and then Taq DNA polymerase is responsible for primer extension (Figure 1B). The addition of highfidelity DNA polymerase does not affect the release of fluorescent signal mediated by Taq DNA polymerase.

Our previous study demonstrated that $0.15 \mathrm{U}$ high-fidelity DNA polymerase per $25 \mu \mathrm{l}$ reaction was the optimal amount for the proof-reading function to remove mismatched bases, but not to compete

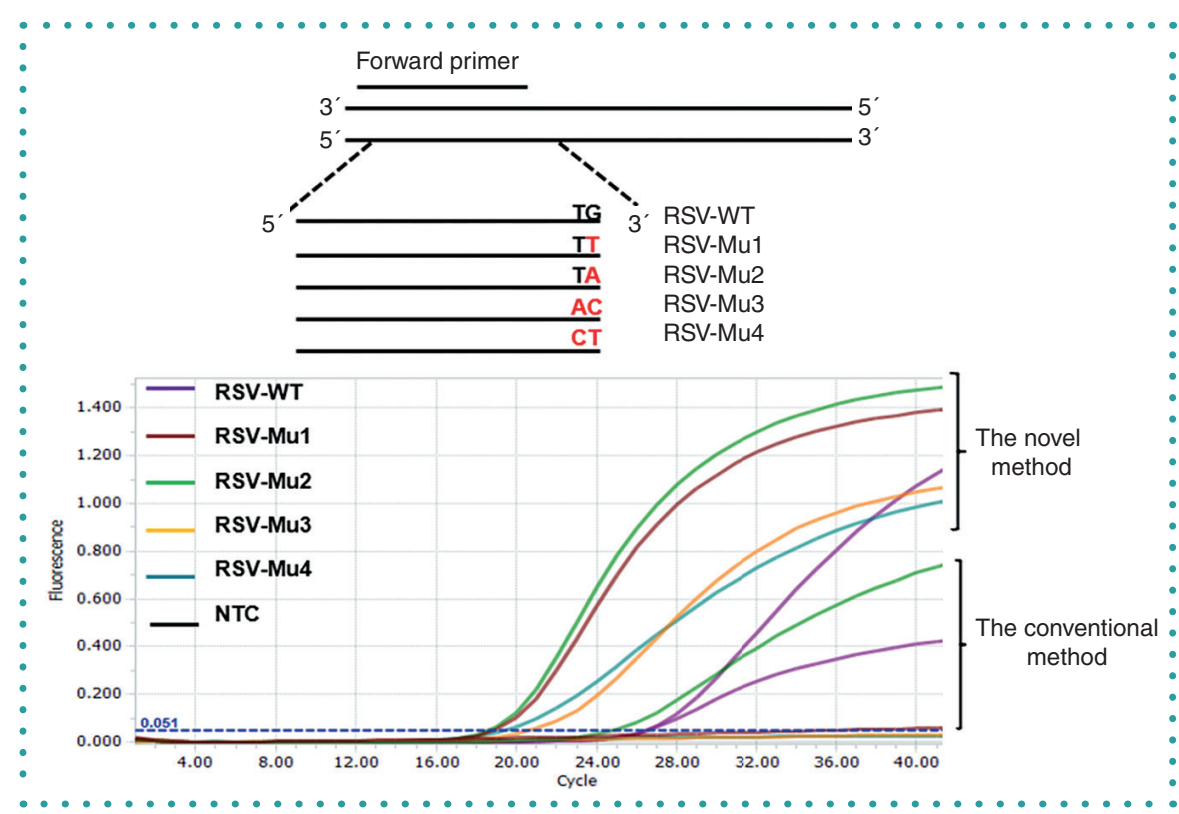

Figure 3. Flexibility of the new RSV RT-qPCR assay. WT: Wild-type; Mu: Mutant; NTC: No template control.

with Taq DNA polymerase for primer extension [32]. To determine the optimal amount of high-fidelity DNA polymerase in the mismatch-tolerant RT-qPCR, different amounts (from 0 to $1 \mathrm{U}$ ) of Q5 high-fidelity DNA polymerase were added into the $25 \mu \mathrm{l}$ qPCR reaction system with $1 \mathrm{U}$ of Taq DNA polymerase to amplify mutant templates. The best amplification results were obtained when $0.15 \cup$ Q5 high-fidelity DNA polymerase was added (data not shown). Increased amount of high-fidelity DNA polymerase reduced the PCR amplification efficiency possibly due to the competition between both high-fidelity DNA polymerase and Taq DNA polymerase. With $0.15 \mathrm{U}$ Q5 high-fidelity DNA polymerase, the amount of Taq DNA polymerase was further optimized at $0.75 \mathrm{U}$ per $25 \mu \mathrm{l}$ reaction. The optimal $25 \mu \mathrm{l}$ reaction mix with $0.75 \mathrm{U}$ of Taq and $0.15 \mathrm{U}$ of Q5 DNA polymerases was used in subsequent experiments.

\section{Sequence analysis of RSV-A \& -B strains} To analyze the inter- and intra-variability of two RSV groups, we downloaded all available RSV-A and -B sequences from the GenBank database. A total of 636 sequences were obtained, including 435 RSV-A and 201 RSV-B genomic sequences. Sequence analyses showed that RSV-A and -B shared low genetic similarity (approximately 47.8\%). In particular, RSV-A had lower intra-similarity
(73.5\%) than RSV-B (97.5\%), indicating RSV-B has more genetic variation than RSV-A. Therefore, to develop a new RSV RT-qPCR assay for simultaneous detection of RSV-A and RSV-B, degenerate primers are needed. In this study, we used a pair of degenerate primers and a minor groove binder probe that were previously described [23]. To evaluate the primers and probe, we analyzed the sequence features of all RSV strains in the positions of the primers and probe. The results showed that RSV-A and -B have very different sequence features in those positions (Figure 2). The main sequence difference between RSV-B and RSV-A occurs near the $3^{\prime}$ end of the forward primer (Figure 2), which provides an explanation for why most methods had low detection efficiency for RSV-B strains. Furthermore, there are some RSV-A variants carrying one or more mutations at the $3^{\prime}$ end of the forward primer, implying a possibility of missed detection and false negative results.

\section{Flexibility of the mismatch-tolerant RT-qPCR}

To assess the performance of the novel mismatch-tolerant RT-qPCR assay, we constructed four RSV mutants that form one to two mismatches with the forward primer at the 3 ' end (Figure 3). For the wild-type, the novel method had a smaller threshold cycle (Ct) value compared with the conventional 


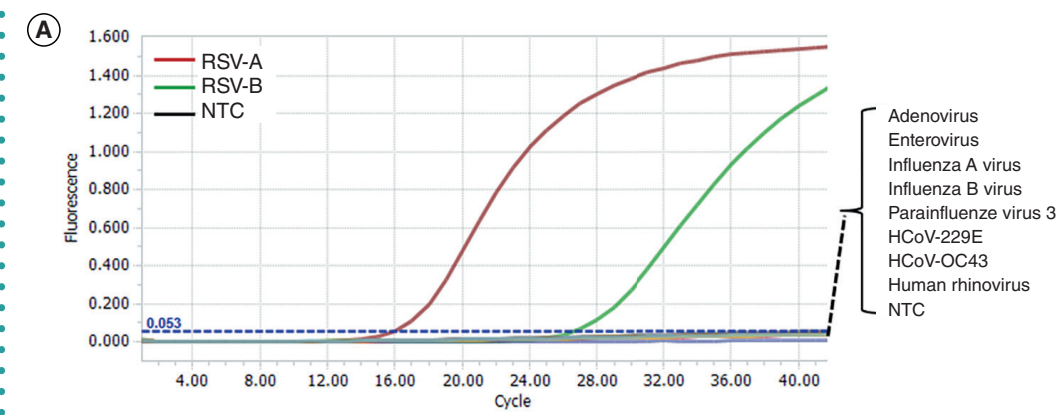

(B)

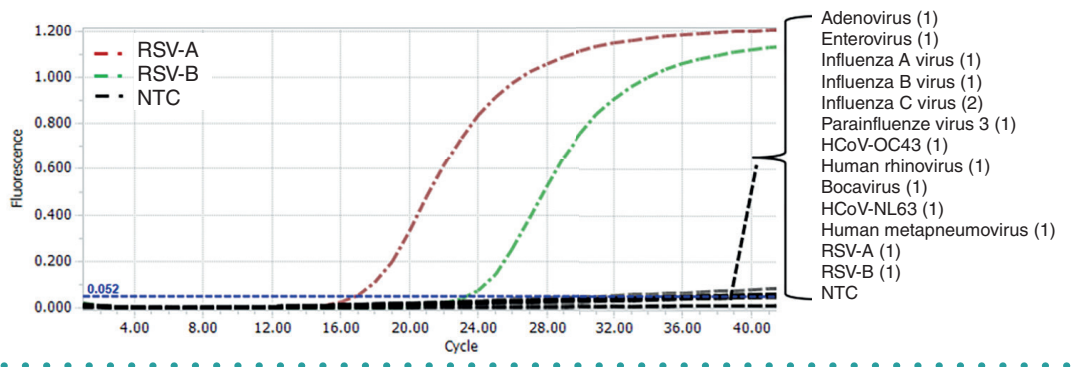

Figure 4. Specificity of the new RSV RT-qPCR assay. (A) Using standard virus strains. (B) Using clinical samples. A total of 14 positive clinical samples for RSV-A, RSV-B, adenovirus, enterovirus, influenza-A, $-\mathrm{B}$, and $-\mathrm{C}$ viruses, parainfluenza virus $3, \mathrm{HCoV}-\mathrm{OC} 43, \mathrm{HCoV}-\mathrm{NL} 63$, human rhinovirus, bocavirus and human metapneumovirus, were used. NTC: No template control. (n): The number of positive clinical samples.

$\checkmark$ method, and generated substantially higher fluorescent intensity (1.31 vs 0.46 ; $\mathrm{p}<0.01$, paired T-Test). Importantly, we found that the novel mismatch-tolerant RT-qPCR assay could amplify all four mutants (Ct: 17.07 to 21.62), while the conventional method failed to amplify almost all mutants except mutant 2 (RSV-Mu2) (Ct: 25.97) (Figure 3). Slightly higher amplification efficiency was observed for the templates forming one mismatch at the $3^{\prime}$ end of the primer than for those forming two mismatches by the novel assay, indicating that more mismatches at the $3^{\prime}$ end of the primer had stronger inhibitory effect on amplification. In spite of this, compared with the conventional RT-qPCR assay, the novel mismatch-tolerant RT-qPCR assay generated not only lower $\mathrm{Ct}$ values, but also higher fluorescent intensity, indicating that the novel assay largely improves the amplification efficiency of QPCR for templates carrying mismatches.

We then asked whether the qPCR assay with only high-fidelity DNA polymerase can also tolerate the mismatches between primer and template well, and whether the novel mismatch-tolerant RT-qPCR is also valid for other viruses. To answer the two questions, we performed an assay with only high-fidelity DNA polymerase, and compared it with the novel mismatchtolerant and the conventional qPCR assays. We tested two viruses (RSV and
Table 1. Limit of detection of the novel mismatch-tolerant qPCR.

RSV standard (PFU/ml) Positive results/total tested Positive percentage (\%)

\begin{tabular}{|l|l|l|}
\hline $1 \times 10^{4}$ & $10 / 10$ & 100 \\
\hline $1 \times 10^{3}$ & $10 / 10$ & 100 \\
\hline $1 \times 10^{2}$ & $10 / 10$ & 100 \\
\hline $1 \times 10^{1}$ & $10 / 10$ & 100 \\
\hline 5 & $10 / 10$ & 100 \\
\hline 2.5 & $10 / 10$ & 100 \\
\hline 1 & $5 / 10$ & 50 \\
\hline
\end{tabular}

HCoV-229E), and found that the assay with only high-fidelity DNA polymerase had similar performance to the novel mismatchtolerant RT-qPCR (with a mix of Taq and high-fidelity DNA polymerases) for RSV, both of which are better than the conventional $\mathrm{qPCR}$, but with worse performance than the novel RT-qPCR assay and the conventional qPCR for HCoV 229E (Supplementary Figure $\mathrm{S} 1$ ). The results suggested the assay with only high-fidelity DNA polymerase has low amplification stability for mutants forming mismatches, and is not suitable for wide application. Tests for bocavirus further demonstrated the wide applicability of the novel mismatchtolerant RT-qPCR for virus detection (Data not shown)

\section{Specificity \& sensitivity}

To determine the specificity of the novel assay, a total of eight other respiratory virus reference strains were tested together with RSV-A and -B strains. Except for the two RSV strains, no positive results were observed for any other non-RSV respiratory virus (Figure 4A). We further used 14 positive clinical samples for RSV-A, RSV-B and 11 other respiratory viruses to test the specificity of the novel assay. No amplification was observed for all 12 positive clinical samples for non-RSV respiratory viruses (Figure 4B). The results indicated that the new assay is highly specific to RSV.

Sensitivity testing showed that when the template input was more than $10 \mathrm{PFU} /$ ml RSV RNA, all 10 reactions (100\%) had positive amplification. When the template input was $1 \mathrm{PFU} / \mathrm{ml}$ RSV RNA, five of the 10 reactions were positive (Table 1). The LOD of the new RSV RT-qPCR assay was calculated to be $1.8 \mathrm{PFU} / \mathrm{ml}$.

\section{Evaluation of the novel RSV assay using} 40 clinical samples

The standard curve shows a strong linear relationship $\left(R^{2}=0.99\right)$ between log RSV $\mathrm{RNA} P F U / \mathrm{ml}$ and $\mathrm{Ct}$ values with a dynamic scope from $1 \times 10^{0}$ to $1 \times 10^{5} \mathrm{PFU} / \mathrm{ml}$ (Figure 5A). To evaluate the novel RSV RT-qPCR method, we used 40 clinical samples from children with ARTIs and compared the novel assay with the conventional method. 20 samples were detected as positive, and the others as negative by both methods. Although linear correlation 
for 20 positive samples was observed between the novel assay and the conventional method $\left(R^{2}=0.99\right)$, $C t$ values generated by the novel assay appeared to be slightly lower (implying higher copy number) than those by the conventional method (Figure 5B), suggesting that the novel assay was slightly more sensitive than the conventional method.

To further evaluate the performance of the new method for RSV detection, we compared it with a commercial kit, the Huayin RSV RNA kit, using the same 40 clinical samples. 20 positive samples as shown by the novel assay were also positive according to the commercial kit, demonstrating a $100 \%$ consistency (Table 2). The correlation coefficient $\left(R^{2}\right)$ between both methods was 0.69 (Figure $5 \mathrm{C}$ ). The $95 \%$ limits of agreement between the two methods was estimated to be within the range of -0.7 to $+1.34 \log (\mathrm{PFU} / \mathrm{ml})$ by BlandAltman analysis (Figure 5D).

Importantly, we found that 16 (80\%) of 20 positive samples had higher viral copy numbers according to the novel method compared with the Huayin RSV RNA kit, and another four samples showed opposite results (Figure $5 \mathrm{C}$ ). In particular, there were two samples (Sp19 and Sp16) with obvious higher copy numbers according to the novel method compared with the commercial kit, and this was speculated to be the result of mismatches between primers and viral variants. To address this hypothesis, we sequenced the targeted RSV genomic regions from samples Sp19 and Sp16, as well as another two samples (Sp8 and Sp9) that had slightly lower copy numbers according to the novel method. As expected, Sp16, Sp19 and Sp8 carried G-A and C-T mutations in the primer and probe regions, respectively (Figure 5E), which formed a mismatch with the 3 ' end of the forward primer, and a mismatch with the third site of the $3^{\prime}$ end of the probe (Figure 5E). An additional mismatch at the $3^{\prime}$ end of the reverse primer was observed in sample Sp19. There were five mutations in the sample Sp9 to form mismatches with the forward primer. Further phylogenetic analysis showed that RSV strains from the samples Sp16, Sp19 and Sp8 belonged to RSV-A, and that in the sample Sp9 was RSV-B (Supplementary Figure S2). These results indicated that the novel
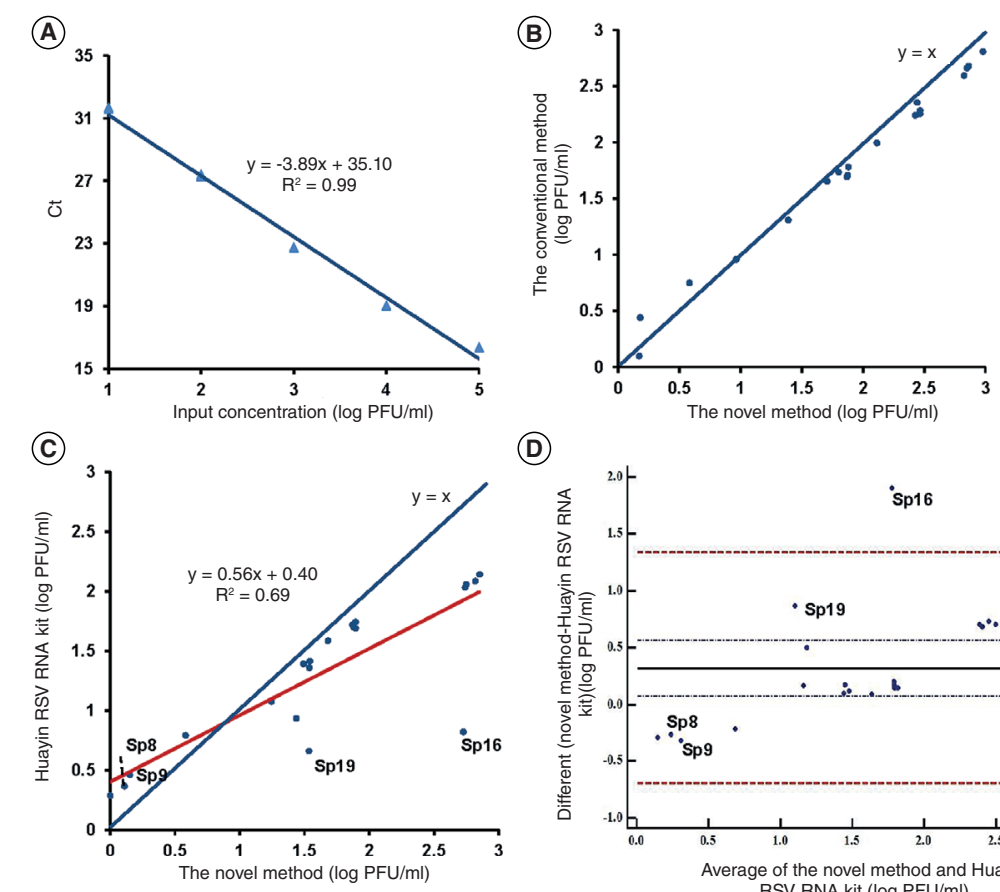

(D)

(E)
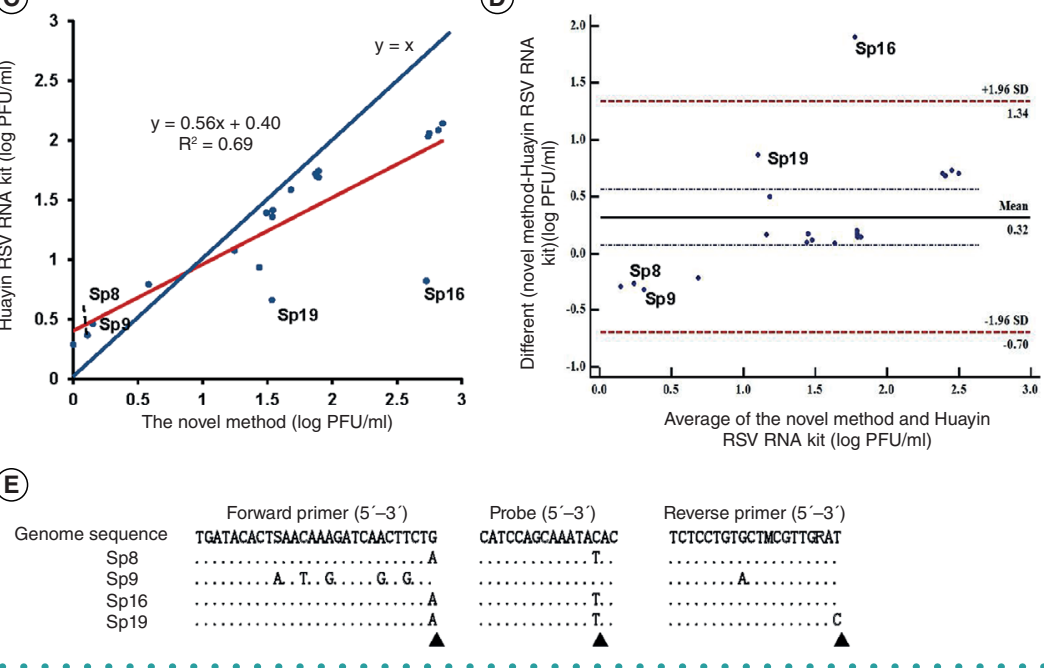

Figure 5. Comparison of the novel RSV assay with the conventional method and a commercial kit in RSV detection of 40 clinical samples. (A) The standard curve of the novel RSV assay. (B) Comparison between the novel and the conventional assays. The diagonal line is shown in the plot. (C) Comparison between the novel RSV assay and a commercial RSV detection kit (Huayin, China). (D) Bland-Altman plot to assess the agreement between the novel assay and the kit for 20 RSV-positive clinical samples. (E) Sequence analysis of primer and probe regions of several special RSV strains in clinical samples.

method has high capacity to amplify virus variants forming mismatches to primers or probe.

Viruses, especially RNA viruses, have higher mutation rates and a faster replication cycle than bacteria and fungi pathogens, and often exist in the form of quasispecies in the human body, implying a large viral population of intra-host variants $[5,6]$. Current qPCR methods have low power to efficiently detect various virus variants [33]. In this study, we developed a

\begin{tabular}{|c|c|c|c|}
\hline & & \multicolumn{2}{|c|}{ The Novel RSV assay } \\
\hline & & Positive & Negative \\
\hline The Commercial RSV & Positive & 20 & 0 \\
\hline RNA detection kit & Negative & 0 & 20 \\
\hline
\end{tabular}

mismatch-tolerant qPCR method by simply adding a small amount of high-fidelity DNA polymerase into the conventional TaqMan qPCR system. The novel method tolerates mismatches between primers and template well, and exhibits high efficiency to amplify virus variants. Therefore, the mismatch-tolerant qPCR represents a simple, sensitive and promising approach to replace conventional qPCR for accurate and sensitive detection of highly variable viruses. 


\section{- FUTURE PERSPECTIVE}

This work provides a mismatch-tolerant qPCR technique that can tolerate mismatches between primers and templates well, and is especially suited to detect various virus variants. Use of this method in multiple systems will be particularly helpful in the diagnostics of infectious diseases. Droplet digital PCR is a promising technique that enables precise and absolute quantitation of nucleic acids without requiring a calibration curve. However, the presence of virus variants forming mismatches with primers may result in failure in DNA amplification in some miniemulsion droplets, thereby causing an underestimate of actual nucleic acid copy number in a given sample. This new method can be of use in droplet digital PCR assays, and improve their accuracy in absolute nucleic acid quantification. Furthermore, loopmediated isothermal amplification (LAMP) is a promising tool for diagnostics of infectious diseases in resource-limited settings. A similar principle to the mismatch-tolerant qPCR is recommended to be used in the LAMP assay by adding a small amount of high-fidelity DNA polymerase to improve the applicability of LAMP to highly variable viruses.

\section{AUTHOR CONTRIBUTIONS}

CZ conceived and designed the study. YL carried out the experiments. $\mathrm{CZ}, \mathrm{YL}, \mathrm{ZW}, \mathrm{YH}$, $\mathrm{YZ}$ and $\mathrm{QC}$ analyzed data. $\mathrm{CZ}, \mathrm{YL}, \mathrm{ZW}$ and $\mathrm{YH}$ interpreted the results. $\mathrm{CZ}$ and $\mathrm{ZW}$ contributed reagents and materials. $C Z$ and YL wrote the manuscript. All authors read and approved the final manuscript.

\section{COMPETING \& FINANCIAL INTERESTS DISCLOSURE}

This work was funded by grants from the National Science and Technology Major Project of China (2017ZX10103009-002 and 2018ZX10101004003001), and Shanghai Sailing Program (16YF1412500). The authors have no other relevant affiliations or financial involvement with any organization or entity with a financial interest in or financial conflict with the subject matter or materials discussed in the manuscript apart from those disclosed.

No writing assistance was utilized in the production of this manuscript.

\section{ETHICAL CONDUCT OF RESEARCH}

The use of patients' samples in this research was approved by the Ethics Committees of Shanghai Public Health Clinical Center. Oral or written informed consent was obtained from childrens' parents or guardians before enrollment.

\section{SUPPLEMENTARY DATA}

To view the supplementary data that accompany this paper please visit the journal website at: www.future-science. com/doi/suppl/10.2144/btn-2018-0184

\section{REFERENCES}

1. Mehand MS, Al-Shorbaji F, Millett P, Murgue B. The WHO R\&D Blueprint: 2018 review of emerging infectious diseases requiring urgent research and development efforts. Antiviral Res. 159, 63-67 (2018).

2. Fenollar $F$, Mediannikov 0 . Emerging infectious diseases in Africa in the 21 st century. New Microbes and New Infections 26, S10-S18 (2018).

3. Van Doorn HR. Emerging infectious diseases. Medicine 42(1), 60-63 (2014).

4. Kubista M, Andrade JM, Bengtsson $\mathrm{M}$ et al. The real-time polymerase chain reaction. Mol. Aspect. Med. 27(2-3), 95-125 (2006)

5. Drake JW. The distribution of rates of spontaneous mutation over viruses, prokaryotes, and eukaryotes. Ann. NY Acad. Sci. 870(1), 100-107 (1999).

6. Domingo E, Perales C. Quasispecies and virus. Eur. Biophys. J. 47(4), 443-457 (2018).

7. Sanjuan R, Nebot MR, Chirico N, Mansky LM, Belshaw R. Viral mutation rates. J. Virol. 84, 9733-9748 (2010).

8. Holmes EC. What does virus evolution tell us about virus origins? J. Virol. 85, 5247-5251 (2011).

9. Phaneuf $\mathrm{CR}, \mathrm{Oh} \mathrm{K}, \mathrm{Pak} \mathrm{N}$ et al. Sensitive, microliter PCR with consensus degenerate primers for Epstein Barr virus amplification. Biomed. Microdevice. 15(2), 221-231 (2013).

10. Jindal $N$, Chander $Y$, De Abin $M$ et al. Amplification of four genes of influenza $A$ viruses using a degenerate primer set in a one step RT-PCR method. J. Virol. Methods 160(1-2), 163-166 (2009).

11. Lefever S, Pattyn F, Hellemans J, Vandesompele J. Single-nucleotide polymorphisms and other mismatches reduce performance of quantitative PCR assays. Clin. Chem. 59(10), 1470-1480 (2013)

12. Borchers AT, Chang C, Gershwin ME, Gershwin LJ. Respiratory syncytial virus-a comprehensive review. Clin. Rev. Allergy Immunol. 45(3), 331-379 (2013).

13. Byington $\mathrm{CL}$, Wilkes J, Korgenski K, Sheng X. Respiratory syncytial virus-associated mortality in hospitalized infants and young children. Pediatrics 135(1), e24-e31 (2015).

14. Pickles RJ, Devincenzo JP. Respiratory syncytial virus (RSV) and its propensity for causing bronchiolitis. $J$. Pathol. 235(2), 266-276 (2015).

15. Nair H, Nokes DJ, Gessner BD et al. Global burden of acute lower respiratory infections due to respiratory syncytial virus in young children: a systematic review and meta-analysis. Lancet 375(9725), 1545-1555 (2010).
16. Allen KE, Beekmann SE, Polgreen $P$ et al. Survey of diagnostic testing for respiratory syncytial virus (RSV) in adults: Infectious disease physician practices and implications for burden estimates. Diagnostic Microbiol. Infect. Dis. 92(3), 206-209 (2018)

17. Shi T, Mcallister DA, O'Brien KL et al. Global, regional, and national disease burden estimates of acute lower respiratory infections due to respiratory syncytial virus in young children in 2015: a systematic review and modelling study. Lancet 390(10098), 946-958 (2017).

18. Caballero MT, Polack FP. Respiratory syncytial virus is an 'opportunistic' killer. Ped. Pulmonol. 53(3), 664-667 (2018).

19. Mufson MA, Orvell C, Rafnar B, Norrby E. Two distinct subtypes of human respiratory syncytial virus. J. Gen. Virol. 66(10), 2111-2124 (1985).

20. Dewhurst-Maridor G, Simonet V, Bornand JE, Nicod LP, Pache JC. Development of a quantitative TaqMan RT-PCR for respiratory syncytial virus. J. Virol. Methods 120(1), 41-49 (2004)

21. Essaidi-Laziosi M, Lyon M, Mamin A, Rocha MF, Kaise L, Tapparel C. A new real-time RT-qPCR assay for the detection, subtyping and quantification of human respiratory syncytial viruses positive- and negative-sense RNAs. Virol Methods 35, 9-14 (2016).

22. Abdel-Moneim AS, Shehab GM, Alsulaimani AA, AlMalky MIR. Development of TaqMan RT-qPCR for the detection of type $A$ human respiratory syncytial virus. Mol. Cell. Probes 33, 16-19 (2017).

23. You HL, Chang SJ, Yu HR, Li CC, Chen CH, Liao WT. Simultaneous detection of respiratory syncytial virus and human metapneumovirus by one-step multiplex real-time RT-PCR in patients with respiratory symptoms. BMC Pediatrics 17(1), 89 (2017).

24. Hu A, Colella M, Tam JS, Rappaport R, Cheng SM. Simultaneous detection, subgrouping, and quantitation of respiratory syncytial virus $A$ and $B$ by real-time PCR. J. Clin. Microbiol. 41(1), 149-154 (2003).

25. Thornton HV, Blair PS, Lovering AM, Muir P, Hay AD. Clinical presentation and microbiological diagnosis in paediatric respiratory tract infection: a systematic review. Br. J. Gen. Prac. 65(631), e69-e81 (2015).

26. Mahony J, Chong S, Bulir D, Ruyter A, Mwawasi $K$ Waltho D. Development of a sensitive loop-mediated isothermal amplification assay that provides specimen-to-result diagnosis of respiratory syncytial virus infection in 30 minutes. J. Clin. Microbiol. 51(8), 2696-2701 (2013)

27. Mu Y, Zeng J, Chen Q et al. New method for the visual detection of human respiratory syncytial virus using reverse transcription loop-mediated amplification. $J$. Virol. Methods 206, 84-88 (2014)

28. Eboigbodin KE, Moilanen K, Elf S, Hoser M. Rapid and sensitive real-time assay for the detection of respiratory syncytial virus using RT-SIBA(R). BMC Infect. Dis. 17(1), 134 (2017)

29. Dong W, Chen Q, Hu Y et al. Epidemiological and clinical characteristics of respiratory viral infections in children in Shanghai, China. Arch. Virol. 161(7), 1907-1913 (2016).

30. Liu J, Mu Y, Dong W et al. Genetic variation of human respiratory syncytial virus among children with feve and respiratory symptoms in Shanghai, China, from 2009 to 2012. J. Mol. Epidemiol. Evol. Genetics Infect. Dis. 27, 131-136 (2014).

31. Bi W, Stambrook PJ. Detection of known mutation by proof-reading PCR. Nucleic. Acids. Res 26(12), 3073-3075 (1998)

32. Hao W, Fan L, Chen Q et al. Modified proofreading PCR for detection of point mutations, insertions and deletions using a ddNTP-blocked primer. PLoS One 10(4), e0123468 (2015)

33. Zhang M, Liu K, Hu Y et al. A novel quantitative PCR mediated by high-fidelity DNA polymerase. Sci. Rep. 7(1), 10365 (2017) 